\title{
Beamforming Aided-SSK Modulation for MIMO System with Energy Harvesting
}

\author{
Zhenxing Yang ${ }^{+}$, Tianyu Lu, Xiaojuan Yin \\ Nanjing University of Posts and Telecommunications, China
}

\begin{abstract}
In contrast to conventional preprocessing aided spatial modulation (PSM), which carries partial information using the indices of receive antennas, we exploit one receive antenna to implicitly convey information and meanwhile harvest energy at the remaining antennas. Based on this, we propose two novel beamforming schemes. The first scheme is to maximize the sum energy harvested by the receiver. And the second scheme is to maximize the minimum receiving power on each antenna except for the antenna whose index conveys information. A closed form solution and an iterative algorithm are given, respectively. Simulation results demonstrate that proposed two schemes can harvest a certain amount of energy with nearly same achievable rate. But the second scheme is superior to the first scheme and PSM scheme in terms of bit error rate (BER) performance.
\end{abstract}

Keywords: Space shift keying (SSK), simultaneous wireless information and power transfer (SWIPT), transmitter zero forcing (TZF).

\section{Introduction}

Spatial Modulation (SM) technology has drawn intensive attention since it has been proposed for its higher spectral efficiency and lower system complexity. In contrast to conventional MIMO technologies, for SM technology, only one transmit antenna is activated and a single-radio-frequency (RF) is required each time. Therefore inter-antenna synchronization at the transmitter and inter-channel interference can be completely avoided [1].

Similarly, as a novel MIMO technique, SSK modulation can be considered to be a simplified form of spatial modulation, which only employs the indices of the transmit antennas to convey information. The technique takes advantage of the differences in the signals received from different transmit antennas to distinguish the transmitted information messages. In [2], a pragmatic communication strategy for the use of SSK modulation in two amplify-and-forward relaying is proposed. In the strategy, the transceiver employs SSK modulation to send the signal and detects the signal based on the maximal-ratio combining principle.

Different from SSK modulation, conventional SM conveys information both by the indices of the transmit antennas and by modulation symbol. At the transmitter, the transmission data streams are divided into two parts. One part is transmitted by employing conventional phase shift keying (PSK)/quadrature amplitude modulation (QAM) and the other part is implicitly transmitted by activating a single transmit antenna. [3] considers a generalized SM with multiplexing (GSMM), where multiple transmit antennas are activated. They presented a general capacity analysis encompassing different forms of SM accompanied by tight lower and upper bounds of the achievable rate. In [9], the authors proposed a novel scheme, in which, spatial modulation works in both phase and quadrature parts of received signals. A virtual spatial modulation

\footnotetext{
+ Corresponding author.

E-mail address:yangzx92@qq.com
} 
scheme that performs index modulation on virtual parallel channels is proposed in [10]. This scheme conveys information through both the indices of virtual parallel channels and the M-ary modulated symbols.

More recently, a new transmission scheme, which is called receive SM (RSM) is proposed in [4]. By assuming channel state information (CSI) is known at transmitter, the transmit power is focused only on one receive antenna selected according to the transmitted information. This can be realized with the help of transmitter zero forcing (TZF) preprocessing. Furthermore, [5] studied the secrecy mutual information of PSM for multi-antenna eavesdropper wiretap channel. The precoding design achieves a good tradeoff of the performance between Bob and Eve via maximizing the power received at Bob while simultaneously minimizing the power received at Eve.

Though there are many papers about various forms of SM, but only few papers combine SM with energy harvesting. A new concept of an energy pattern aided SWIPT system is proposed in [6], where in addition to power transfer, information is conveyed by the specific receive antenna indices to which the power is delivered as well as by the conditional modulation symbol. In [7], a wireless-powered communication (WPC) system is considered where a wireless device (WD) communicates to an information receiver (IR) with the harvested radio frequency (RF) energy from a wireless energy transmitter (WET). The idea is that an antenna of the WD sends information to IR and at the same the rest of the antenna harvests energy from the WET.

Inspired by [6] and [7], a SSK-MIMO simultaneous wireless information and power transfer (SWIPT) system is considered. We attempt to only use the index of one receive antenna to convey information, and simultaneously utilize the rest antennas to harvest energy. This can be achieved by forcing the transmit power of the selected antenna to zero and allocating total power to other receive antennas. Then we propose two sub-optimal beamforming schemes to maximize correct detection probability of the selected antenna index. The first scheme is to optimize the total harvested energy, and the second scheme is to maximize the minimum receiving power on each antenna apart from the antenna carrying information. To solve the optimization problem of two schemes, a close form optimal solution is given for the first scheme and an iterative algorithm is presented for the second scheme. Simulation results demonstrate that though the first scheme can harvest more energy than the second scheme with approximately equal achievable rate, but the BER performance of the second scheme outperforms the first scheme and PSM scheme.

The remainder of this paper is organized as follows. In sectionII, the system model and an optimization formulation for the problem of beamforming design is explained. In section III, two beamforming schemes are proposed. The simulation results are presented in section IV. The paper is summarized in section V.

Notations: $|\bullet|\|\bullet\|(\bullet)^{T}(\bullet)^{H} \operatorname{rank}(\bullet)$ and $\operatorname{Tr}(\bullet)$ denote the absolute value, Frobenius norm, transpose, Hermitian transpose, 'rank' and determinant, respectively. For a Hermitian matrix $\mathbf{A}, \lambda_{\max }(\mathbf{A})$ is its maximal eigenvalue. Furthermore, $P(\bullet)$ represents the occurrence probability of an event.

\section{System Model and Problem Formulation}

Consider a MIMO system. It is assumed that the receiver has $N_{r}\left(=2^{m}, m=1,2, \ldots\right)$ antennas and the transmitter is equipped with $N_{t}\left(\geq N_{r}\right)$ antennas. The indices of receiver's $N_{r}$ receive antenna are used to carry $\mathrm{m}$ bits in principles of SSK. According to the transmitted information, the i-th receive antenna is selected to carry the information. At the receiver, we intend to decode information from the index of ith receive antenna and harvest energy from the remaining antennas.

Assume that the channels from transmitter to receiver experience flat Rician fading, with the channel matrix expressed as $\mathbf{H}=\left[\mathbf{h}_{1}, \mathbf{h}_{2}, \ldots, \mathbf{h}_{N r}\right]^{T}$, where $\mathbf{h}_{i}$ is a $N_{t}$-dimensional column vector containing the complex channel gains from transmitter's $N_{t}$ antennas to receiver's i-th receive antenna.

We assume linear transmit precoding at the transmitter. Thus at the receiver, the received signal can be formulated as

$$
\mathbf{y}=\mathbf{H w}_{i} x+\mathbf{n}
$$

where $\mathbf{w}_{i} \in \mathcal{C}^{N_{t} \times 1}$ is the beamforming vector and $x$ is an energy-carrying signal, while $\mathbf{n}$ is a complex Gaussian noise vector distributed with zero mean and a covariance matrix $\sigma_{n}^{2} \mathbf{I}_{N_{r}}$, expressed as 
$\mathbf{n} \sim \mathcal{C N}\left(0, \sigma_{n}^{2} \mathbf{I}_{N_{r}}\right)$ for convenience. Since $x$ carries no information, it can be any arbitrary random signal provided that its power spectral density satisfies certain regulations microwave radiation. In this paper, we assume that $x$ is chosen from a uniform distribution sequence with $x x^{H}=1$.

To decode information from the index of ith antenna and simultaneously harvest energy as much as possible from the other antennas, the transmit power on the ith antenna which conveys information is forced to zero. Thus transmitter employs TZF preprocessing to generate beamforming vector $\mathbf{w}_{i}$, which satisfies

$$
\mathbf{h}_{i}^{T} \mathbf{w}_{i}=0
$$

Ignoring the noise term of equation (1), the total energy harvested by the receiver is given by

$$
\Upsilon=\eta \rho\left\|\mathbf{H w}_{i}\right\|^{2}
$$

In which $\eta \in(0,1]$ is the energy conversion efficiency and $\rho$ is the power-split ration at the receiver.

On the other hand, the remaining $1-\rho$ portion of the receive power enters the information transfer path, which is used to decode the transfer information. The signal can be described as

$$
\mathbf{y}_{I}=\sqrt{1-\rho}\left(\mathbf{H w}_{i} x+\mathbf{n}\right)
$$

According to the formula (2), we can further decompose (4) as

$$
\begin{aligned}
& y_{I k}=\sqrt{1-\rho}\left(\mathbf{h}_{k}^{T} \mathbf{w}_{i}+n_{k}\right) \forall k \neq i \\
& y_{I i}=\sqrt{1-\rho} n_{i}
\end{aligned}
$$

Based on (5), we can detect the SSK symbol i easily. The detection algorithm can be described as

$$
\hat{i}=\arg \min _{k \in\left\{1,2, \ldots, N_{r}\right\}}\left\{(1-\rho)\left|y_{I k}\right|^{2}\right\}
$$

From (6) we can know that the detection complexity is $\mathcal{O}\left(2^{m}\right)$ Since the value of $\rho$ does not affect the SSK symbol dectection based on (6), the correct detection probability can be formulated as

$$
\begin{aligned}
P_{c} & =P\left(\left|y_{I i}\right|^{2}<\min _{k \neq i}\left|y_{I k}\right|^{2}\right) \\
& =P\left(\left|n_{i}\right|^{2}<\min _{k \neq i}\left|\mathbf{h}_{k}^{T} \mathbf{w}_{i}+n_{k}\right|^{2}\right) \\
& =\prod_{k \neq i} P\left(\left|n_{i}\right|^{2}<\left|\mathbf{h}_{k}^{T} \mathbf{w}_{i}+n_{k}\right|^{2}\right)
\end{aligned}
$$

The third equation of (7) holds due to the independence of noise.

The beamforming vector can be designed to maximize the correct detection probability subject to transmit power constraint $P_{t}$ and formula (2) constraint. The optimization problem can be described as

$$
\begin{array}{ll}
\text { P0: } & \max _{\mathbf{w}_{i}} P_{c} \\
\text { s.t. } & \mathbf{h}_{i}^{T} \mathbf{w}_{i}=0 \\
& \left\|\mathbf{w}_{i}\right\|^{2}=P_{t}
\end{array}
$$

From (7), we can know that it is difficult to derive a simple closed-form expression of the correct detection probability $P_{c}$, so the problem (8) is very hard to solve. Therefore two sub-optimal beamforming schemes are proposed in next section.

\section{Beamforming Design Schemes}

According to (7), the correct detection probability increases as the power on each receive antenna except for the $\$ \mathrm{i} \$$-th increases. Based on this, in this section, we put forward two sub-optimal beamforming schemes 
that maximize the correct detection probability. For the first scheme, a closed form optimal solution is given in sub-section A. In sub-section B, an iterative algorithm is presented to obtain the optimal solution to the second scheme.

\subsection{Maximizing the Sum Energy Harvested by Receiver}

In this sub-section, our aim is to maximize the weighted power transferred to all receive antennas subject to the transmit power constxaints and TZF constraint. Denote $\alpha_{k} \geq 0(\forall k)$ as the given energy weight for each antenna. Define $\Gamma=\sum \eta \rho \alpha_{k}\left|\mathbf{h}_{k}^{T} \mathbf{w}_{i}\right|^{2}$ as the objective function. Especially we set $\alpha_{k}=1(\forall k)$, and then the objective function ${ }^{k}{ }^{1}$ quite the total harvested energy. In this way, the optimization problem can be described as follows.

$$
\begin{aligned}
& \mathrm{P} 1: \max _{\mathbf{w}_{i}} \Upsilon \\
& \text { s.t. } \mathbf{h}_{i}^{T} \mathbf{w}_{i}=0 \\
&\left\|\mathbf{w}_{i}\right\|^{2}=P_{t}
\end{aligned}
$$

Let $\mathbf{V}_{i}^{0}$ is a unitary matrix formed by the orthogonal basis of the null space of vector $\mathbf{h}_{i}^{T}$. Therefore beamforming vector $\mathbf{w}_{i}$ can be formed as a linear combination of the columns of $\mathbf{V}_{i}^{0}$, given by

$$
\mathbf{w}_{i}=\mathbf{V}_{i}^{0} \mathbf{r}_{i}
$$

where $\mathbf{r}_{i}$ is a $\left(N_{t}-1\right)$-dimensional arbitrary vector satisfying $\left\|\mathbf{r}_{i}\right\|^{2}=P_{t}$.

Upon applying (10), (9) can be reformulated with respect to $\mathbf{r}_{i}$ as

$$
\mathbf{r}_{i}^{*}=\underset{\left\|\mathbf{r}_{i}\right\|^{2}=P_{t}}{\arg \max } \eta \rho \mathbf{H} \mathbf{V}_{i}^{0} \mathbf{r}_{i} \|^{2}
$$

When applying the singular value decomposition (SVD) on $\left(\mathbf{H} \mathbf{V}_{i}^{0}\right)^{H} \mathbf{H} \mathbf{V}_{i}^{0}$, we obtain

$$
\left(\mathbf{H} \mathbf{V}_{i}^{0}\right)^{H} \mathbf{H} \mathbf{V}_{i}^{0}=\mathbf{U}_{i} \boldsymbol{\Sigma}_{i} \mathbf{U}_{i}^{H}
$$

Then we can easily get $\mathbf{r}_{i}^{*}={ }_{H} \sqrt{P_{t}} \mathbf{U}_{i}^{1}$, where $\mathbf{U}_{i}^{1}$ is the eigenvector corresponding to the largest eigenvalue of the matrix $\left(\mathbf{H V}_{i}^{0}\right)^{H} \mathbf{H} \mathbf{V}_{i}^{0}$. So the optimal solution of problem (P1) can be given as $\mathbf{w}_{i}^{*}=\mathbf{V}_{i}^{0} \mathbf{r}_{i}^{*}=\sqrt{P_{t}} \mathbf{V}_{i}^{0} \mathbf{U}_{i}^{1}$.

\subsection{Maximizing the Minimum Received Power of Each Antenna}

Since we cannot find a beamforming vector that make all $\left|\mathbf{h}_{k}^{T} \mathbf{w}_{i}\right|^{2}(\forall k \neq i)$ largest, accordingly in this sub-section, we propose a sub-optimal scheme that makes the correct detection probability relatively large. In this scheme, we aim to maximize the worst received power. Then the optimization problem can be formulated as

$$
\begin{gathered}
\mathrm{P} 2: \max _{\mathbf{w}_{i}} \min _{k \neq i}\left|\mathbf{h}_{k}^{T} \mathbf{w}_{i}\right|^{2} \\
\text { s.t. } \mathbf{h}_{k}^{T} \mathbf{w}_{i}=0 \\
\left\|\mathbf{w}_{i}\right\|^{2}=P_{t}
\end{gathered}
$$

Applying formula (10), the problem P2 can be reformulated as follows.

$$
\mathrm{P} 2-1: \max _{\left\|\mathbf{r}_{i}\right\|^{2}=P_{t}} \min _{k \neq i}\left|\mathbf{h}_{k}^{T} \mathbf{V}_{i}^{0} \mathbf{r}_{i}\right|^{2}
$$

The problem P2-1 is non-convex and difficult to solve directly. Defining $\mathbf{R}_{i}=\mathbf{r}_{i} \mathbf{r}_{i}^{H}$, the problem P2-1 can be rewritten as 


$$
\begin{array}{ll}
\text { P2-2: } & \min _{t \in R, \mathbf{R}_{i}}-t \\
\text { s.t. } & \operatorname{Tr}\left(\mathbf{G}_{k} \mathbf{R}_{i}\right) \geq t, \forall k \neq i \\
& \operatorname{Tr}\left(\mathbf{R}_{i}\right)=P_{t} \\
& \mathbf{R}_{i} \geq 0 \\
& \operatorname{rank}\left(\mathbf{R}_{i}\right)=1
\end{array}
$$

where $\mathbf{G}_{k}=\left(\mathbf{h}_{k} \mathbf{V}_{i}^{0}\right)^{H} \mathbf{h}_{k} \mathbf{V}_{i}^{0}$ and $t$ is an additional slack variable.

However the problem P2-2 is still non-convex due to the rank constraint. Reference [8], a semidefinite relaxation problem (SDP) can be given as follows.

$$
\begin{array}{ll}
\mathrm{P} 2-3: \min _{t \in R, \mathbf{R}_{i}}-t+\mu\left\lfloor P_{t}-\lambda_{\max }\left(\mathbf{R}_{i}^{(\kappa)}\right)\right. \\
& \left.-\operatorname{Tr}\left(\mathbf{r}_{i}^{(\kappa)} \mathbf{r}_{i}^{(\kappa) H}\left(\mathbf{R}_{i}-\mathbf{R}_{i}^{(\kappa)}\right)\right)\right] \\
\text { s.t. } \quad & \operatorname{Tr}\left(\mathbf{G}_{k} \mathbf{R}_{i}\right) \geq t, \forall k \neq i \\
& \operatorname{Tr}\left(\mathbf{R}_{i}\right)=P_{t} \\
& \mathbf{R}_{i} \geq 0
\end{array}
$$

where $\mu>0$ is a sufficiency large penalty factor and $\mathbf{r}_{i}^{(\kappa)}$ is a unit-norm eigenvector corresponding the maximum eigenvalue of the feasible solution $\mathbf{R}_{i}^{(\kappa)}$. Then an algorithm is given to generate iterative solution $\mathbf{R}_{i}^{(\kappa+1)}$ for the ultimate solution of P2-2.

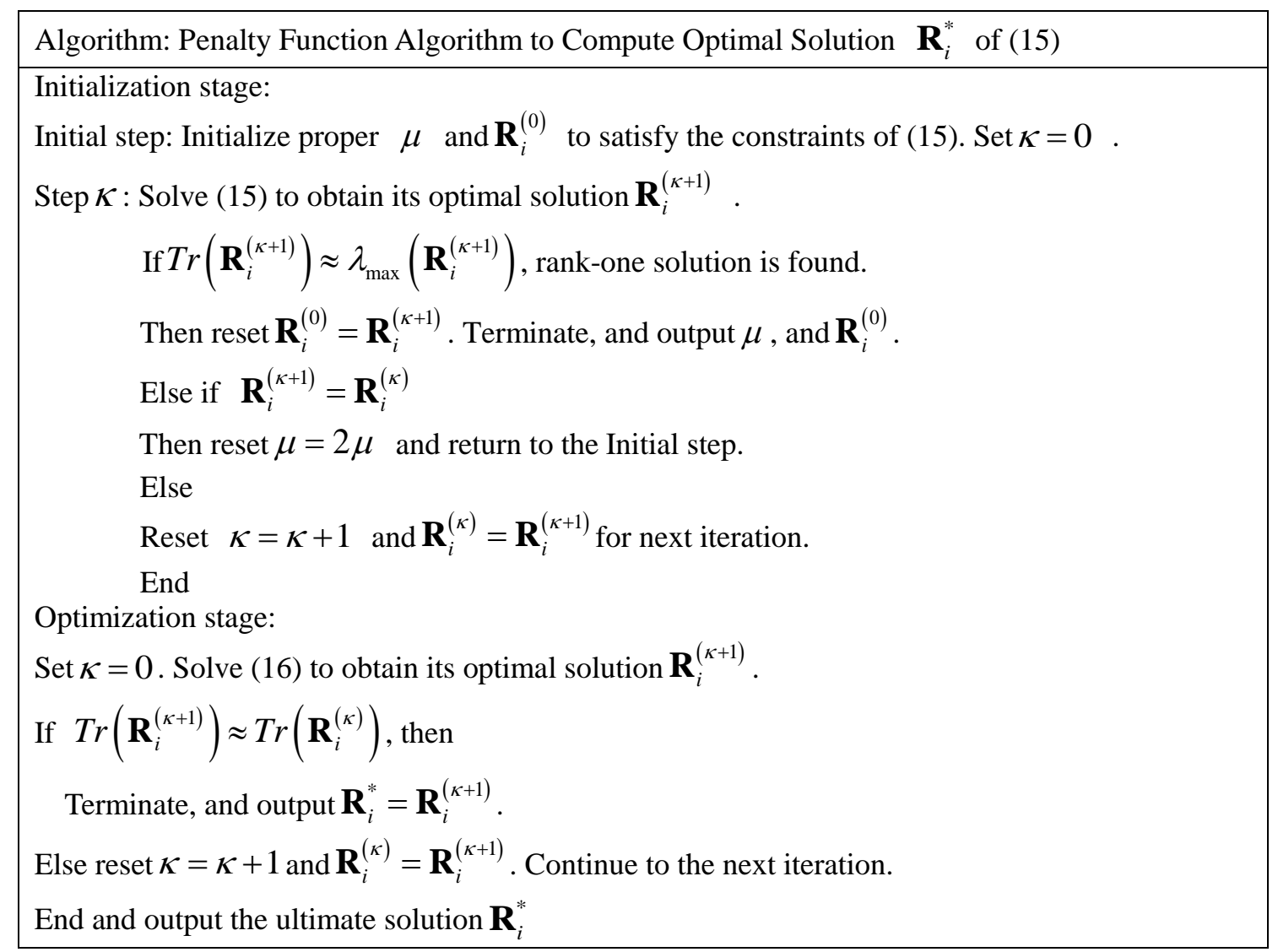

Applying the iterative algorithm we can obtain the optimal solution $\mathbf{R}_{i}^{*}$ of (15). Then the optimal solution admits the singular value decomposition 


$$
\mathbf{R}_{i}^{*}=\mathbf{r}_{i}^{*} \mathbf{r}_{i}^{* H}
$$

Therefore we can get the optimal solution of (13) via $\mathbf{w}_{i}^{*}=\mathbf{V}_{i}^{0} \mathbf{r}_{i}^{*}$.

\section{Simulation Results}

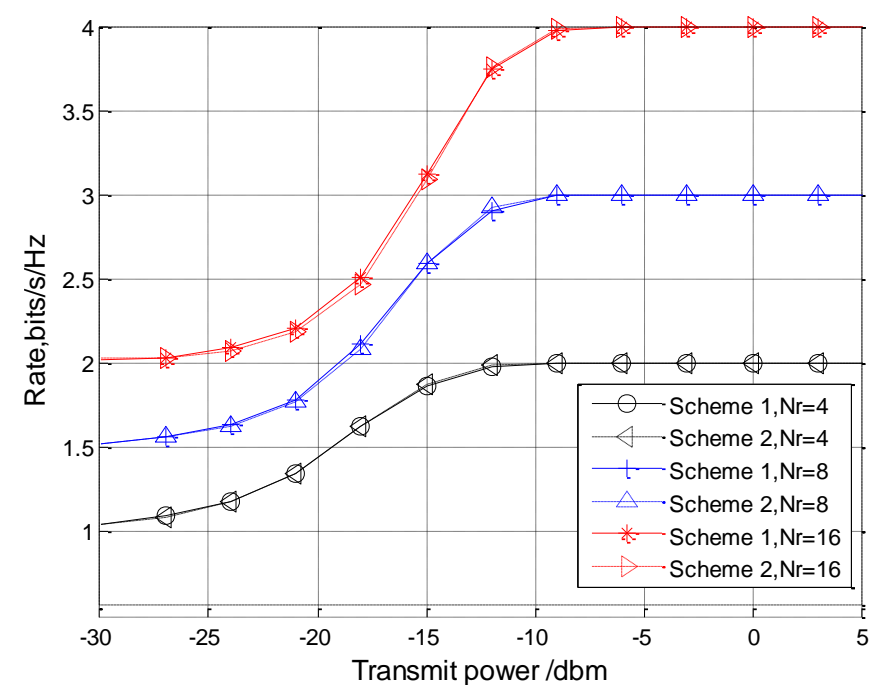

Fig. 1: The rate achieved by proposed schemes.

In this section, the performance of the proposed beamforming schemes for a MIMO system is demonstrated in terms of achievable rate, BER and the energy harvested by the receiver. The number of transmit antennas is set $N_{t}=32$ and the receiver antenna number is $N_{r}=4,8,16$ respectively. We assume that the signal attenuation from the transmitter to the receiver is $20 \mathrm{~dB}$ corresponding to an equal distance of 1 meter. The channel matrix is generated from Rician fading channel. In the following simulation, we set Rician factor $K=10$, noise power $\sigma_{n}^{2}=-40 \mathrm{dBm}$, the power-split ration $\rho=0.9$, and energy conversion efficiency $\eta=0.8$, respectively.

Fig. 1 compares the information rate achieved by the receiver versus different transmit power constraint. As expected, when the noise power is constant, with the increase of the transmission power, the receiver finally reaches the upper bound rate of $\log _{2} N_{r}$, which are 2,3,4 bits/s/Hz when the number of the receiver antennas is $4,8,16$, respectively. It can be seen clearly that the rates obtained by different schemes are approximately equal.

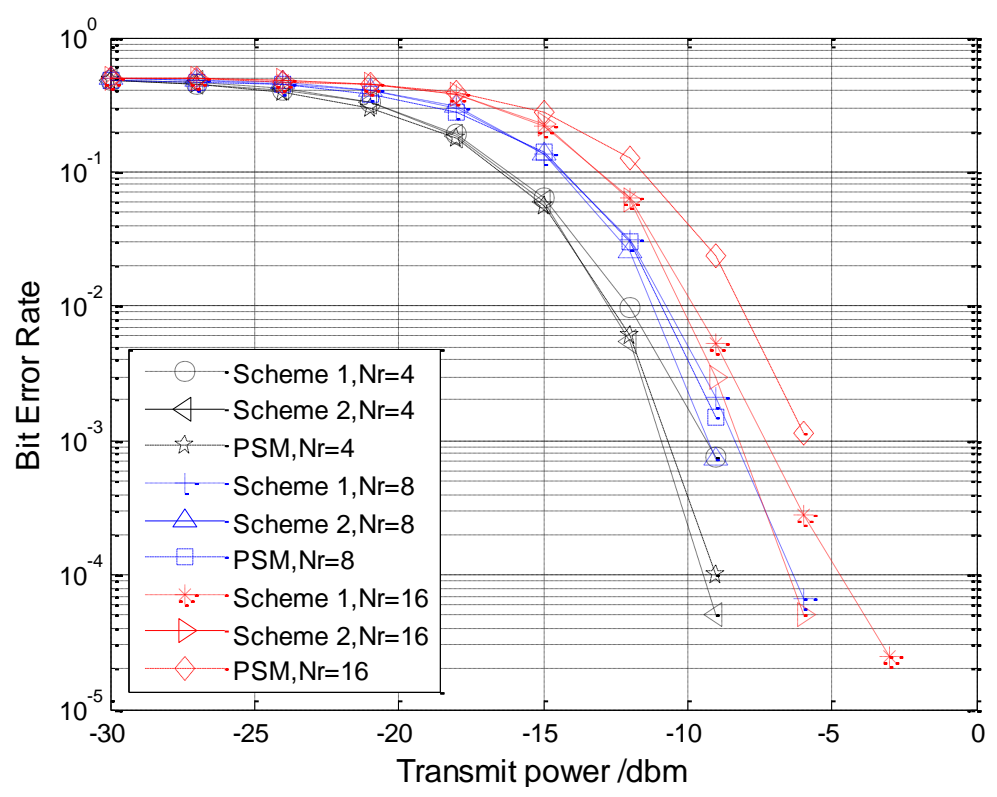

Fig. 2: The BER performance of proposed schemes and PSM scheme. 
Fig. 2 compares BER performance. Fig. 2 shows the differences between proposed schemes and PSM scheme that focuses the transmit power only on one receive antenna. Clearly, the BER performance of the three schemes is approximately the same at small transmit power. While at large transmit power, the BER performance of the second scheme is better than the first scheme and PSM scheme. This is because that in the second scheme the beamforming vector is designed to approximately minimize BER, so the second scheme performs best among three schemes. Then making a comparison between the first scheme and PSM scheme, we find that scheme one performs worse than PSM scheme when the number of receive antennas is equal to 4 and 8 respectively. But a better performance can be achieved by the first scheme than PSM scheme as soon as the number of receive antennas increases to 16 .

A comparison between the proposed schemes in terms of the harvested energy is presented in fig.3, where transmit power is fixed to $30 \mathrm{dBm}$. The figure demonstrates an increase in the sum harvested energy with increase in the number of receive antennas. It is observed that the first scheme outperforms the second scheme in terms of the sum harvested energy as expected. What's more, it can be seen clearly that the harvested energy gaps between two schemes are about $0 \mathrm{dbm}, 0.2035 \mathrm{dBm}, 0.3738 \mathrm{dBm}, 0.4563 \mathrm{dBm}$, respectively. Therefore the gap becomes larger as the number of receive antennas increases.

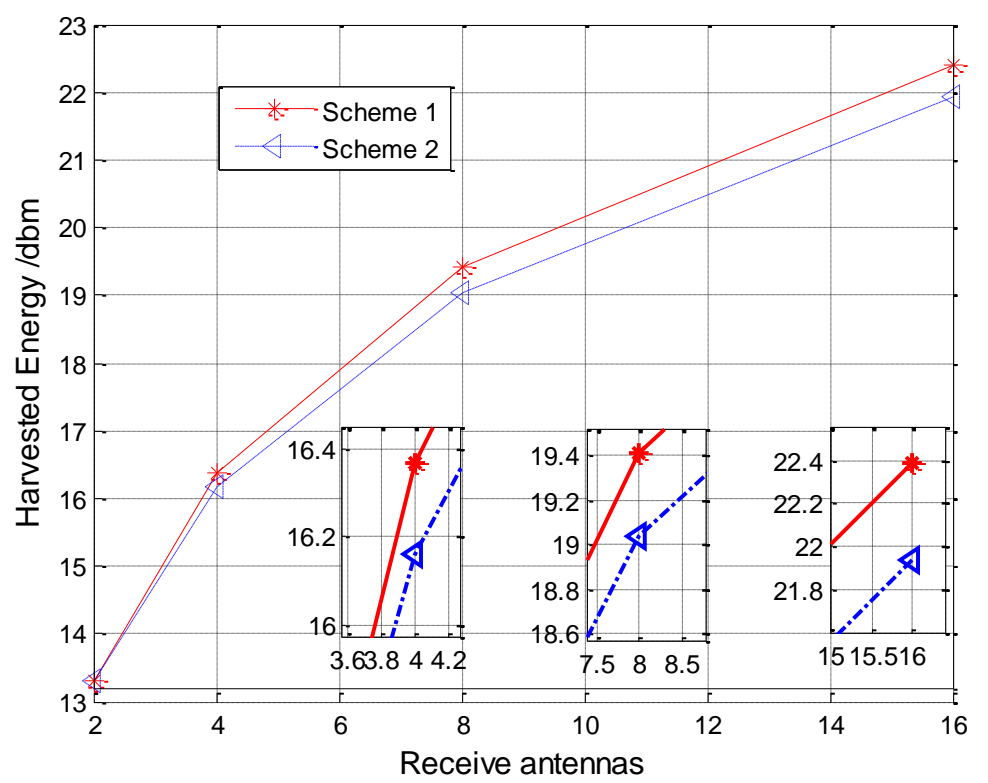

Fig. 3: The differences of total harvested energy between proposed schemes.

\section{Conclusion}

In this letter, we have proposed a novel idea to combine PSM with energy harvesting. Based on this idea, two beamforming schemes are proposed. The corresponding maximization problems are formulated and optimally solved. Finally, a closed form solution and an iterative algorithm are given, respectively. The achievable rate, error performance and harvested energy have been studied. Our studies show that both schemes can obtain a certain amount of energy without marked achievable rate differences. Meanwhile the second scheme outperforms the first scheme and PSM scheme in terms of BER performance.

\section{References}

[1] M. Di Renzo, H. Haas, A. Ghrayeb, S. Sugiura, and L. Hanzo, "Spatial modulation for generalized mimo: Challenges, opportunities, and implementation," Proceedings of the IEEE, vol. 102, no. 1, pp. 56-103, Jan 2014.

[2] M. Wen, X. Cheng, H. Vincent Poor, B. Jiao, "Use of SSK Modulation in Two-Way Amplify-and-Forward Relaying," IEEE Trans. on Veh. Technol. March 2014, Vol. 63, no. 3, pp. 1498-1504.

[3] Ahmad A. I. Ibrahim, Taejoon Kim, David J. Love. "On the Achievable Rate of Generalized Spatial Modulation Using Multiplexing Under a Gaussian Mixture Model," IEEE Transaction on Communications, April, 2016, Vol. 64, No. 4, pp. 1588-1599. 
[4] L.-L. Yang, "Transmitter preprocessing aided spatial modulation for multiple-input multiple-output systems," in Proc. IEEE 73rd VTC Spring, May 2011, pp. 1-5.

[5] F. Wu, R. Zhang, L.-L. Yang, W. Wang, "Transmitter Precoding-Aided Spatial Modulation for Secrecy Communications," IEEE Transactions on Vehicular Technology, Jan. 2015, pp. 467-471.

[6] R. Zhang, L-L. Yang, L. Hanzo, "Energy Pattern Aided Simultaneous Wireless Information and Power Transfer," IEEE Journal on Selected Areas in Communications, Jan. 2015, vol. 33, no. 8, pp. 1492-1504.

[7] M. Zhang, X. Cheng, "Spatial-Modulation-Based Wireless-Powered Communication for Achievable Rate Enhancement," IEEE Commun. Lett. , Mar. 2017, pp. 1365-1368.

[8] A. H. Phan, H. D. Tuan, H. H. Kha, D. T. Ngo, "Nonsmooth Optimization for Efficient Beamforming in Cognitive Radio Multicast Transmission,” IEEE Transaction on Signal Processing, March 2012, pp. 2941-2951.

[9] Jun Li, Miaowen Wen, Xiang Cheng, Yier Yan, Sangseob Song, Moon Ho Le“Generalised precoding-aided quadrature spatial modulation,” IEEE Trans. on Veh. Technol. May 2017, vol. 66, no.2, pp.1881-1886.

[10] Jun Li, Miaowen Wen, Meng Zhang, Xiang Cheng, "Virtual spatial modulation," IEEE Access, 2016, vol. 4, pp. 6929-6938. 